\title{
Transplantation of lymphocytes and immune lymphocytes in sarcoidosis and in malignant lymphadenomas
}

\author{
J. KOHOUT \\ M.D. \\ Wilhelminenspital, Second Medical Service, Vienna, Austria
}

\begin{abstract}
Summary
When lymphocytes of a homologous donor are transferred intracutaneously to another person a local reaction of delayed type is seen at the site of transfer 24-48 hr later ('normal lymphocyte transfer reaction'). When sensitized lymphocytes of the recipient afterwards are transferred back to the donor in normal cases the local reaction is much stronger ('immune lymphocyte transfer reaction'). When the recipients were patients with acute sarcoidosis or with Hodgkin's disease of stage III and IV, the normal lymphocyte transfer reaction was considerably weaker than in normal cases. The reaction of patients with chronic sarcoidosis was similar to normal controls, likewise the reaction of early cases of Hodgkin's disease. In back transfer of sensitized lymphocytes of above mentioned recipients to the donors the immune lymphocyte transfer reaction was stronger in acute sarcoidosis than in stage III of Hodgkin's disease, but always weaker than in normal cases. The reactions of patients with chronic sarcoidosis were similar to normal controls. The investigations show a parallel to the findings with passive transfer of delayed type sensitivity.
\end{abstract}

\section{Introduction}

Brent and Medawar (1963) showed that, when in guinea-pigs homologous lymphocytes of a donor are injected intradermally to a recipient, a local reaction of delayed type is seen after $24-48 \mathrm{hr}$ at the site of injection. The reaction was named 'normal lymphocyte transfer (NLT) reaction' and suggested as a 'graft versus host' reaction. There is a close correlation between the degree of the NLT reaction and the behaviour of a skin graft when transplanted from the injected animal back to the lymphocyte donor.

When sensitized lymphocytes of the recipient are transferred back to the donor, the local reaction is much stronger than the NLT reaction. As it is released by sensitized 'immune lymphocytes' this reaction was named 'immune lymphocyte transfer (ILT) reaction'.

When the lymphoreticular system is depressed, as after application of X-rays, the NLT and ILT reactions are diminished.

The NLT reaction was studied in man by Gray and Russel (1963), Bridges, Nelson and McGeown (1964) and Goldsmith (1965) especially for histocompatibility tests.

In man, the lymphoreticular system and delayed type sensitivity are depressed especially in malignant lymphadenomas and in sarcoidosis. Aisenberg (1966) found that in Hodgkin's disease the transfer of homologous lymphocytes is followed by a diminished NLT reaction. Therefore it was of interest to investigate: (1) the NLT reaction in patients with sarcoidosis, where depression of delayed type sensitivity is known to occur, and its correlation to the NLT reaction of healthy controls, patients with Hodgkin's disease and tuberculosis; (2) the ILT reaction in the same subjects, showing the ability of a new immune response.

\section{Methods}

\section{Normal lymphocyte transfer reaction (NLT)}

Donors were healthy persons, not older than 50 years. Recipients were patients with acute and chronic sarcoidosis, Hodgkin's disease, tuberculosis, and healthy controls. Donors and recipients did not previously receive blood transfusions or corticosteroids.

Lymphocyte concentrates were made as follows. Venous blood of the donor to which heparin has been added is put into long test tubes $(25 \times 300 \mathrm{~mm})$ and is divided into plasma and erythrocyte sediment by hatching it for $30 \mathrm{~min}$ at $37^{\circ} \mathrm{C}$ in dextran (mol. wt $250,000,3 \%$ in physiologic saline solution). Plasma rich in leucocytes is then filtered at $37^{\circ} \mathrm{C}$ through cotton filters formed to slightly pressed columns 2-4 $\mathrm{cm}$ high in tiny Pyrex glass tubes $(11 \times 150 \mathrm{~mm})$. Before filtration the cotton is washed with distilled water and then with a nutritive solution (Hanks). Height and firmness of the cotton column is standardized so that $2 \mathrm{ml}$ of plasma filtered through is collected in $50 \mathrm{ml}$ centrifuge tubes containing $500 \mathrm{u}$ of heparin. Fifteen to twenty millititres of plasma may be filtered through one cotton column. Microscopic 
examination of the filtered plasma shows clear and living lymphocytes. Filtered plasma of one donor is collected and concentrated by centrifuging and the sediment thus gained is suspended in Hanks's solution.

The transferred lymphocytes were numbered with $5 \times 10^{6}$ cells. The cells were transferred intracutaneously without delay.

\section{Immune lymphocyte transfer reaction (ILT)}

Donors were the sensitized recipients of the above mentioned NLT reaction (patients with acute and chronic sarcoidosis, Hodgkin's disease, tuberculosis, and healthy controls).

Recipients were the healthy donors of the NLT reaction.

The transfer was made 8 days after the sensitization; the general conditions were the same as in the NLT reaction.

\section{Results}

\section{NLT reaction}

The results of NLT reaction in patients with different diseases and in healthy controls after transfer of homologous lymphocytes of healthy donors (volunteers, hepatitis and other virus diseases were excluded) are to be seen in Fig. 1. The reactions in acute sarcoidosis and in Hodgkin's disease of stages III and IV are weaker than in healthy controls, patients with sarcoidosis in the stage of fibrosis and patients with tuberculosis. Patients with malignant lymphogranulomatosis in an early stage showed a NLT reaction similar to healthy persons.

\section{ILT reaction}

The ILT reactions (Fig. 2) as a rule are much stronger than the NLT reactions in healthy persons and in patients with tuberculosis and chronic sarcoidosis, but are distinctly less strong in acute sarcoidosis. The smallest ILT reactions were found in Hodgkin's disease of stages III and IV, whereas the reactions of cases in an early stage of malignant lymphogranuloma were not essentially different from their NLT reactions.

\section{Discussion}

In lymphocyte transfer reaction a distinct difference can be found between diseases with depression of the lymphoreticular system and others.

Reduced NLT reactions seem to be due to factors in the host. NLT reactions of patients with acute sarcoidosis and with Hodgkin's disease of stage III and IV seem to be diminished to the same extent. The fact that the NLT reaction in chronic sarcoidosis is, as a rule, distinctly stronger than in acute cases suggests that the diminished reaction in acute sarcoidosis is due to another transient process, more or less finished in the stage of fibrosis.

In patients with Hodgkin's disease the NLT reactions in the early stages were nearly normal but in patients in stages III and IV always diminished. This

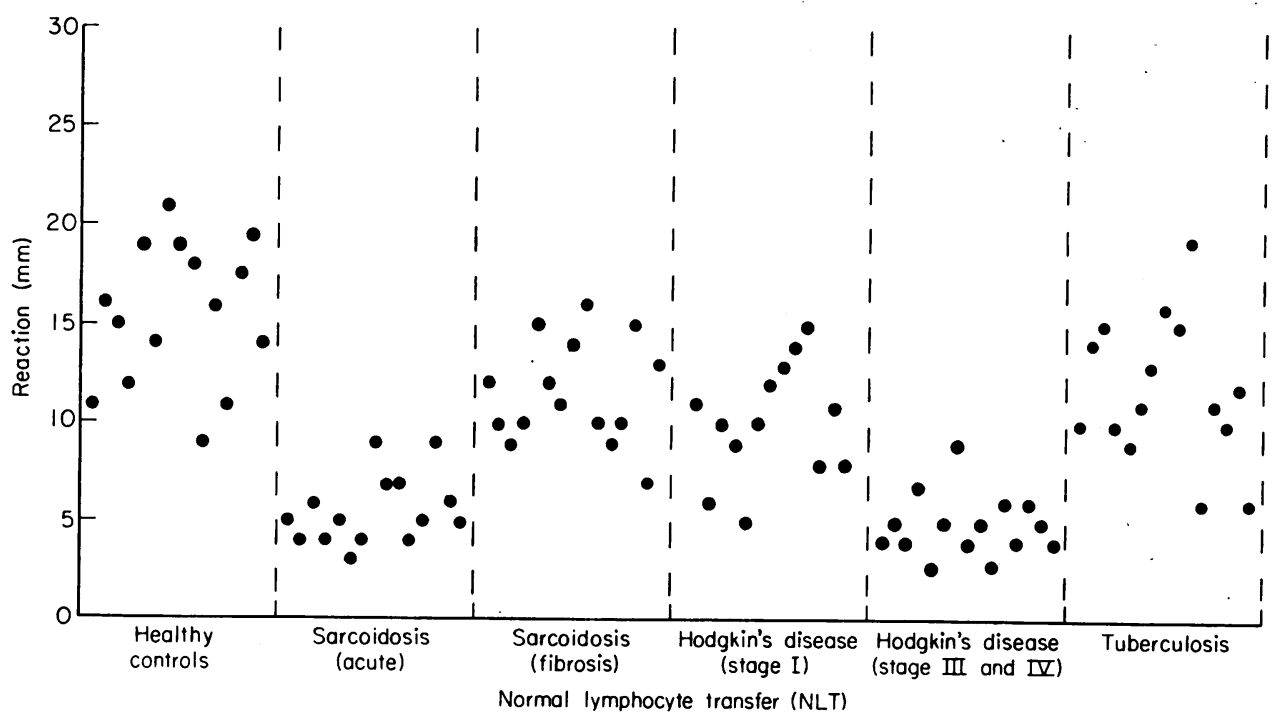

FIG. 1. Transplantation of homologous lymphocytes of healthy donors on patients with different diseases: the NLT reaction is distinctly weaker in cases of acute sarcoidosis and Hodgkin's disease of stage III and IV. Patients with sarcoidosis in stage of fibrosis show a nearly normal reaction. 


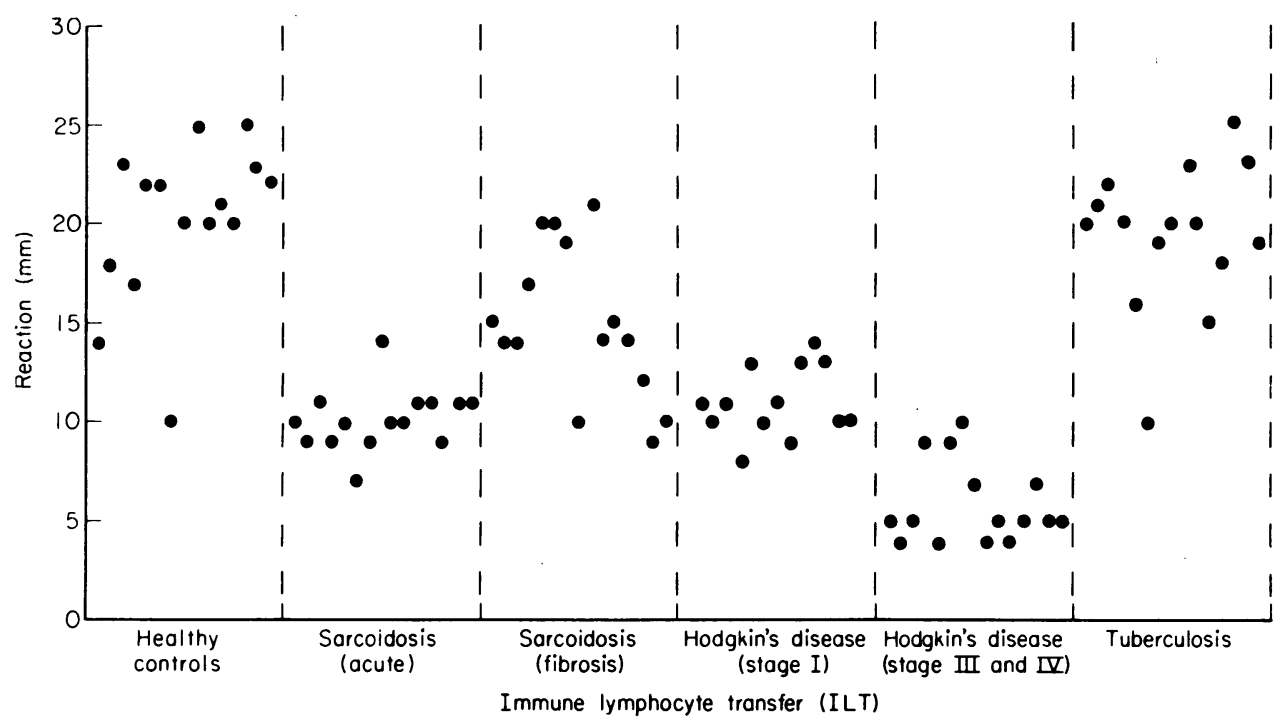

FIG. 2. Transplantation of homologous immune lymphocytes of sensitized recipients with different diseases on the healthy donors: the ILT reactions are essentially stronger than the NLT reactions in control cases and patients with fibrotic sarcoidosis and tuberculosis, but distinctly less strong in acute sarcoidosis. The smallest ILT reactions were found in Hodgkin's disease of stage III and IV. In early stage of malignant lymphogranulomatosis the ILT reactions were not essentially different from their NLT reactions.

different behaviour could be an argument for another mechanism than in sarcoidosis.

Reduced ILT reactions must be due to inherent failure of the lymphocytes to become sensitized. The ILT reactions show more clearly that the immunological deficiency in Hodgkin's disease is greater than in sarcoidosis and that there are also further qualitative differences. The ability to give a new immune response was least in cases of Hodgkin's disease.

The lymphocyte transfer reactions of patients with other diseases show no depression of similar extent.

Both NLT and ILT reaction reduced together would indicate host factors preventing the triggering of lymphocytes.

The investigations with transplantation of lymphocytes and immune lymphocytes show a parallel to the findings with passive transfer of delayed type sensitivity we have reported elsewhere (Kohout, 1967a, b). The passive transfer of delayed type sensitivity is more difficult to accomplish in malignant lymphadenomas than in sarcoidosis and the difference between the stages of disease are the same as we find in the NLT and ILT reaction.

\section{References}

AisenberG, A.D. (1966) Manifestations of immunological unresponsiveness in Hodgkin's Disease. Cancer Research, 26, 1152.

Brent, L. \& Medawar, P.B. (1963) Tissue transplantation: a new approach to the 'typing' problem. British Medical Journal, if, 269.

Bridges, J.M., Nelson, S.D. \& McGeown, M.G. (1964) Evaluation of lymphocyte transfer test in normal and uraemic subjects. Lancet, i, 581.

GolDSMITH, K.L.G. (1965) Donor selection and compatibility typing. British Medical Bulletin, 21, 162.

GRAY, J.G. \& RUSSELl, P.S. (1963) Donor selection in human organ transplantation: a possible screening test. Lancet, ii, 863 .

KоноUт, J. (1967a) Passive transfer of delayed type sensitivity in sarcoidosis. La Sarcoidose. Rapport de la IVe Conférence Internationale, Masson et Cie, Paris, p. 287.

KoHout, J. (1967b) Passive Úberträgung der Tüberkülinallergie bei der Sarkoidose und bei malignen Lymphomen. Wiener klinische Wochenschrift, 79, 795. 